\title{
Psychiatric Symptoms in Temporal Lobe Epilepsy with Left Mesial Hippocampal Sclerosis
}

\author{
Hyun Jang ${ }^{1}$, Sang Hoon $\mathrm{Kim}^{2}$, Sang Hag Park², II Han Choo², and Seung Gon Kim凶 \\ ${ }^{1}$ Department of Psychiatry, Chosun University Hospital, Gwangju, Republic of Korea \\ ${ }^{2}$ Department of Psychiatry, School of Medicine, Chosun University, Gwangju, Republic of Korea
}

A 16-year-old woman was referred to us for depression and persistent suicidal and homicidal ideation. From 2010, the patient visited a neurologist due to recurrent grand mal epilepsy, auditory and visual hallucinations, episodic memory loss, and persistent depression. Upon admission, it was revealed through clinical history taking that she had suffered from chronic bullying from same-sex peers and sexual abuse, twice, from an adult male in the neighborhood when she was 10 years old. A brain magnetic resonance imaging study showed left mesial hippocampal sclerosis. The patient exhibited improvement of her psychiatric symptoms after treatment with a combination of fluoxetine $(30 \mathrm{mg})$ and aripiprazole $(10 \mathrm{mg})$. Children and adolescents with epilepsy experience conflicts in the family, challenges at school, stigma, and psychosocial limitations or deprivations due to their comorbid psychiatric symptoms and hence, psychiatric evaluation and early intervention is important when treating these patients.

Psychiatry Investig 2015;12(2):274-277

Key Words Suicidal ideation, Homicidal ideation, Temporal lobe epilepsy, Left mesial hippocampal sclerosis.

\section{INTRODUCTION}

The prevalence of psychiatric disorders is higher in people with epilepsy than in the general population. In patients with epilepsy, depression occurs in about $30 \%$, anxiety disorders in $10-25 \%$, and psychosis in 2-7\%. ${ }^{1}$ Thus, these patients are more likely to have psychiatric diseases, ${ }^{2}$ and are more than three times as likely to commit suicide as the general population. ${ }^{2,3}$ About $5 \%$ of the adolescents in the community have Major Depressive Disorder (MDD) and another 3.3\% have dysthymia. ${ }^{3}$ Evidence showed that MDD is associated with smaller hippocampal volumes. ${ }^{4-6}$ A previous study found that subjects with depression and a history of childhood abuse had an $18 \%$ smaller mean left hippocampal volume than those never abused, and a $15 \%$ decrease in comparison with healthy subjects. ${ }^{7}$ In identifying teenagers with suicidal tendencies, factors such as a family history of suicide, alcohol and drug abuse, previous

Received: December 20, 2013 Revised: March 31, 2014

Accepted: April 18, 2014 Available online: March 18, 2015

$\bowtie$ Correspondence: Seung Gon Kim, MD, PhD

Department of Psychiatry, School of Medicine, Chosun University, 365 Pilmundaero, Dong-gu, Gwangju 501-717, Republic of Korea

Tel: +82-62-220-3194, Fax: +82-62-225-3659, E-mail: sgkim@chosun.ac.kr

(c) This is an Open Access article distributed under the terms of the Creative Commons Attribution Non-Commercial License (http://creativecommons.org/licenses/by$\mathrm{nc} / 3.0$ ) which permits unrestricted non-commercial use, distribution, and reproduction in any medium, provided the original work is properly cited. suicide attempts, and self-destructive behavior may be valuable. The most significant psychiatric risk factor associated with adolescent suicide is MDD. ${ }^{8}$ Homicide-suicide perpetrators are also often found to suffer from mental illness, with depression being the most common disorder. ${ }^{9}$ In young people with epilepsy, depression and other psychiatric disorders are common but often remain undiagnosed and untreated. Until now, no previous studies have reported a relationship between hippocampal lesions and homicidal or suicidal ideation. Here, we report the case of a 16-year-old woman with temporal lobe epilepsy showing persistent suicidal and homicidal ideation and depressed mood and discuss the efficacy of psychiatric intervention for temporal lobe epilepsy.

\section{CASE}

In August 2013, a 16-year-old woman was referred to us for depression, persistent suicidal and homicidal ideation, episodic memory loss, and personality changes. The patient often forgot what she did, talked about, or when she took a meal. From 2010, the patient visited a neurologist due to recurrent grand mal epilepsy, auditory and visual hallucinations, episodic memory loss, and persistent depression. She underwent electroencephalogram (EEG) assessment, which presented slow or sharp waves and spikes. After taking antiepileptic drugs, the 
symptoms of epilepsy, hallucinations, and episodic memory loss improved and resolved the epileptic waves on EEG, but her depression symptoms became aggravated. Suicidal and homicidal ideations toward her family and schoolmates continued. She showed personality changes such as becoming impatient and isolating herself extremely from peers. In October 2013, although she had psychological counseling for depression, symptoms of repeated self-injurious behaviors and suicidal and homicidal ideations were not improved. In January 2013, neurologists treated her with lamotrigine (up to $200 \mathrm{mg}$ ), but her symptoms did not improve. In August 2013, she was admitted for further evaluation and treatment.

Upon admission, clinical history taking revealed that she had suffered from chronic bullying and victimization from same-sex peers, and had been sexually abuse, twice, by an adult male from her neighborhood when she was 10 years old. On mental state examination (MSE), she endorsed present suicidal and homicidal ideation, depressed mood, repeated nightmares, and flashbacks about bullying or sexual abuse. Routine blood tests, including complete blood count, serum electrolyte, creatinine, glucose, and vitamin $B_{12}$, vitamin $E$, and folate, autoimmune serologies, and electrocardiogram (ECG), chest X-ray, and EEG showed no aberrant findings. However, brain magnetic resonance imaging (MRI) study detected left mesial hippocampal sclerosis (Figure 1).

On the 2nd day, fluoxetine was administered starting from $10 \mathrm{mg}$ up to $40 \mathrm{mg}$. On the 21th day, she showed frequent episodic memory loss at the highest dose. Considering the adverse effect of selective serotonin reuptake inhibitors (SSRIs)

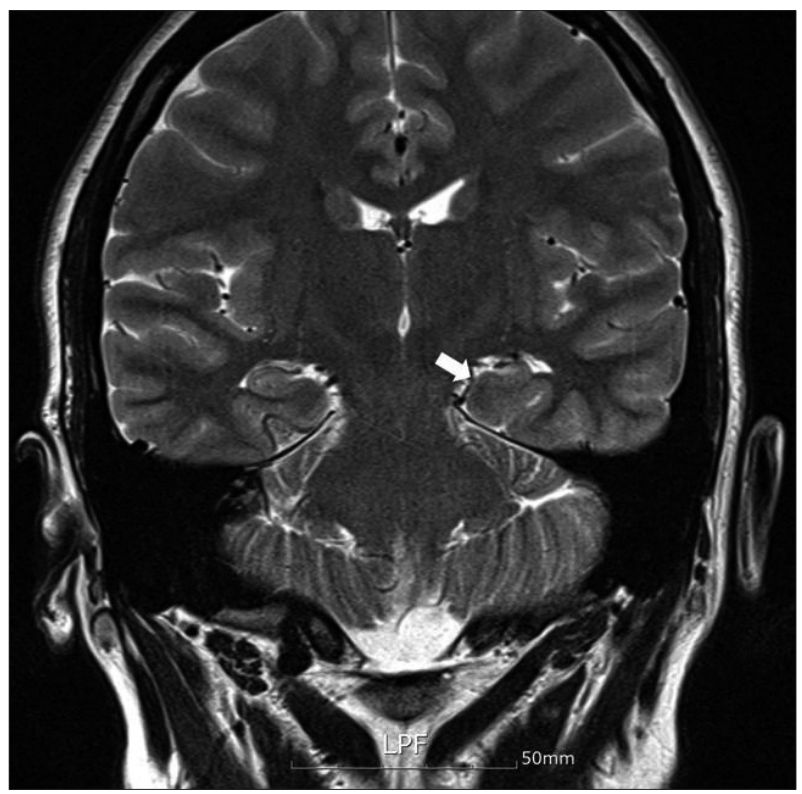

Figure 1. Brain magnetic resonance image (MRI) showing the decrease in size of the left hippocampus with loss of gray-white differentiation. This observation is consistent with mesial temporal sclerosis. such as a decreased seizure threshold, fluoxetine was decreased to $30 \mathrm{mg}$. Aripiprazole was administered starting from $2 \mathrm{mg}$ up to $10 \mathrm{mg}$, to augment the SSRI activity. On the 28th day, she underwent follow-up neuropsychological assessments, which showed improvements in depression, suicidal ideation, and homicidal ideation. She also showed a decrease of episodic memory loss as improvement in depressive symptoms. Upon discharge, the 35th day from admission, most symptoms had significantly improved on follow-up neuropsychological scales (Table 1). After discharge, she regularly visited us as an outpatient and neither depressive symptoms nor suicidal and homicidal ideation appeared again during this period.

\section{DISCUSSION}

In this case report, we present the case of a 16-year-old woman with temporal lobe epilepsy showing persistent suicidal and homicidal ideation, and depressed mood, which improved after taking fluoxetine with aripiprazole augmentation.

The patient presented with temporal lobe epilepsy with left mesial hippocampal sclerosis and psychiatric symptoms including sustained depression, and repetitive suicidal and homicidal ideations. Hippocampal sclerosis describes a pattern of neuronal loss and gliosis involving the medial temporal structures most often encountered in patients with epilepsy. ${ }^{10}$ Evidence has emerged in the past decade that MDD is associated with small hippocampal volumes. ${ }^{4-6} \mathrm{~A}$ previous study reported that children and adolescents with temporal lobe epilepsy had moderate to severe depressive symptoms early in the course of their disease, with an impact on their global functional activities. ${ }^{11} \mathrm{~A}$ previous study found that people with epilepsy were four times more likely to be hospitalized than people without epilepsy, and $30 \%$ had attempted suicide. ${ }^{12}$ Of the suicides committed by people with epilepsy, $81 \%$ to $100 \%$ occur among those with a co-morbid psychiatric disorder, most commonly depression..$^{13}$ Antiepileptic drugs (AEDs), especially barbiturates, are frequently involved in suicides and attempts. ${ }^{14}$ In 2008, the U.S. Food and Drug Administration (US FDA) issued an alert

Table 1. Comparison with neuropsychological tests during admission

\begin{tabular}{lcccc}
\hline & Admission & 14th day & 28th day & Discharge \\
\hline SIQ-Reynolds & 96 & 83 & 61 & 50 \\
SSI-Beck & 32 & 28 & 20 & 14 \\
BDI & 38 & 34 & 22 & 14 \\
HDRS & 34 & 32 & 22 & 16 \\
BHS & 15 & 13 & 9 & 7
\end{tabular}

SIQ-Reynolds: Suicidal Ideation Questionnaire-Reynolds, SSIBeck: 19-item Scale for Suicide Ideation-Beck, BDI: Beck Depression Inventory, HDRS: Hamilton Depression Rating Scale, BHS: Beck Hopelessness Scale 
to healthcare professionals about an increased risk of suicidal thoughts and increased suicidality in people taking AEDs. The overall odds ratio (OR) for spontaneously reported suicidal behavior or ideation among those taking active drugs was 1.8 (95\% CI: $1.24-2.66) .{ }^{15}$ In this case, after the patient took AEDs, her psychiatric symptoms, depression, and suicidal ideation were aggravated, which suggested an association between AEDs and psychiatric symptoms.

In addition, she experienced chronic bullying and physical abuse during childhood and sexual abuse twice at age 10. Childhood sexual abuse is a significant early stressor that may predispose individuals to depression and self-destructive behaviors in adulthood. Previous research found reduced hippocampal volume in victims of psychological trauma with severe sexual or physical abuse in childhood. ${ }^{16}$ Previous suicide attempts, suicidal ideation, and homicidal ideation were also associated with adolescent suicide. ${ }^{8}$ Thus, we considered the association between her psychiatric symptoms, chronic bullying, repeated physical and sexual abuse, and her temporal lobe epilepsy.

The patient showed improvement of psychiatric symptoms in combination treatment with fluoxetine and aripiprazole. Fluoxetine is the only SSRI approved by the FDA for the treatment of depression in patients younger than 18 years of age. ${ }^{17}$ Previous research suggested that reduced serotonin receptor $5-\mathrm{HT}_{1 \mathrm{~A}}$ binding may be related to the increased incidence of depression in patients with epilepsy, due to the reduction of serotonergic neurotransmission in hippocampal epileptic foci. ${ }^{18}$ Thus, SSRIs may be considered for the treatment of depression in adolescents with epilepsy. Antipsychotics are used not only in the treatment of psychotic disorder such as schizophrenia, but also as adjuncts for depressive disorder. Augmentation with atypical antipsychotic agents was significantly more effective than placebo for response and remission. ${ }^{19}$ Aripiprazole received FDA approval for adjunctive therapy in patients with MDD. The product label of aripiprazole recommends a starting dosage of $2-5 \mathrm{mg} /$ day, with a target range of $5-15 \mathrm{mg} /$ day. ${ }^{20} \mathrm{In}$ a previous study, the mean dose at the end of double-blind treatment was $11.8 \mathrm{mg} /$ day, suggesting that the effective dose for patients with major depressive disorder is lower than that recommended for schizophrenia and bipolar disorder. ${ }^{21}$ There was a previous case of a 13-year-old boy on aripiprazole and fluoxetine for depression with persecutory delusions, auditory hallucinations, and a suicide attempt. ${ }^{22}$ In this case, we suggest that the combination of fluoxetine and aripiprazole has therapeutic effects in children and adolescents with temporal lobe epilepsy.

In conclusion, psychiatric symptoms such as depression and suicidal and homicidal ideas are more common in patients with temporal lobe epilepsy. Unfortunately, however, they are often undiagnosed and untreated. Children and adolescents with epilepsy experience acute and chronic stressors such as family conflicts, difficulty in school performance, stigma, and psychosocial limitation or deprivations due to comorbid psychiatric symptoms. Thus, we consider that psychiatric evaluation and early intervention are important for children and adolescents with epilepsy. In the future, longitudinal and prospective studies for the biological underpinnings and treatment of psychiatric symptoms comorbid with temporal lobe epilepsy are needed.

\section{Acknowledgments}

This study was supported by research fund from Chosun University in 2013.

\section{REFERENCES}

1. Gaitatzis A, Trimble MR, Sander JW. The psychiatric comorbidity of epilepsy. Acta Neurol Scand 2004;110:207-220.

2. Harris EC, Barraclough B. Suicide as an outcome for mental disorders. A meta-analysis. Br J Psychiatry 1997;170:205-228.

3. Bell GS, Gaitatzis A, Bell CL, Johnson AL, Sander JW. Suicide in people with epilepsy: how great is the risk? Epilepsia 2009;50:1933-1942.

4. McKinnon MC, Yucel K, Nazarov A, MacQueen GM. A meta-analysis examining clinical predictors of hippocampal volume in patients with major depressive disorder. J Psychiatry Neurosci 2009;34:41-54.

5. Brown ES, Hughes CW, McColl R, Peshock R, King KS, Rush AJ. Association of depressive symptoms with hippocampal volume in 1936 adults. Neuropsychopharmacology 2014;39:770-779.

6. Videbech P, Ravnkilde B. Hippocampal volume and depression: a meta-analysis of MRI studies. Am J Psychiatry 2004;161:1957-1966.

7. Vythilingam M, Heim C, Newport J, Miller AH, Anderson E, Bronen R, et al. Childhood trauma associated with smaller hippocampal volume in women with major depression. Am J Psychiatry 2002;159:2072-2080.

8. Brent DA, Birmaher B. Adolescent depression. New Engl J Med 2002; 347:667-671.

9. Rosenbaum M. The role of depression in couples involved. Am J Psychiatry 1990;147:1036-1039.

10. Blümcke I, Thom M, Wiestler OD. Ammon's horn sclerosis: a mal-developmental disorder associated with temporal lobe epilepsy. Brain Pathol 2002;12:199-211.

11. Pereira A, Valente KD. Severity of depressive symptomatology and functional impairment in children and adolescents with temporal lobe epilepsy. Seizure 2013;22:708-712.

12. Mendez MF, Cummings JL, Benson DF. Depression in epilepsy. Significance and phenomenology. Arch Neurol 1986;43:766-770.

13. Nilsson L, Ahlbom A, Farahmand BY, Åsberg M, Tomson T. Risk factors for suicide in epilepsy: a case control study. Epilepsia 2002;43:644651.

14. Batzel LW, Dodrill CB. Emotional and intellectual correlates of unsuccessful suicide attempts in people with epilepsy. J Clin Psychol 1986;42: 699-702.

15. Arana A, Wentworth CE, Ayuso-Mateos JL, Arellano FM. Suicide-related events in patients treated with antiepileptic drugs. New Engl J Med 2010;363:542-551.

16. Stein MB, Koverola C, Hanna C, Torchia MG, McClarty B. Hippocampal volume in women victimized by childhood sexual abuse. Psychol Med 1997;27:951-959.

17. Bhatia SK, Bhatia SC. Childhood and adolescent depression. Am Fam Physician 2007;75:73-80.

18. Theodore WH, Hasler G, Giovacchini G, Kelley K, Reeves-Tyer P, Herscovitch P, et al. Reduced hippocampal 5HT1A PET receptor binding and depression in temporal lobe epilepsy. Epilepsia 2007;48:1526-1530. 
19. Nelson JC, Papakostas GI. Atypical antipsychotic augmentation in major depressive disorder: a meta-analysis of placebo-controlled randomized trials. Am J Psychiatry 2009;166:980-991.

20. Pae CU, Forbes A, Patkar AA. Aripiprazole as adjunctive therapy for patients with major depressive disorder: overview and implications of clinical trial data. CNS drugs 2011;25:109-127.

21. Berman RM, Marcus RN, Swanink R, McQuade RD, Carson WH, Co-
rey-Lisle PK, et al. The efficacy and safety of aripiprazole as adjunctive therapy in major depressive disorder: a multicenter, randomized, double-blind, placebo-controlled study. J Clin Psychiatry 2007;68:843-853. 22. Preskorn SH. Relating clinical trials to psychiatric practice: part I: the case of a 13-year old on aripiprazole and fluoxetine. J Psychiatr Pract 2003;9:307-313. 